\title{
IL DIRITTO DI MORIRE TRA AUTODETERMINAZIONE TERAPEUTICA, TRASFORMAZIONE DELLA SOVRANITÀ E RIFIUTO SOCIALE DELLA MORTE
}

\author{
CARLO IANNELLO \\ Professore Associato di Istituzioni di Diritto Pubblico \\ Università della Campania «Luigi Vanvitelli» \\ carlo.iannello@unicampania.it
}

\begin{abstract}
RESUMEN: L'articolo analizza la recente ordinanza della Corte costirtuzionale italiana, la n. 207 del 2018, che apre allsa legittimità costituzionale, in determinate circostanze, dell'aiuto al suicidio, letta come indicativa di una generale e recente tendenza volta a dare accoglimento a queste forme di autodeterminazione in un numero sempre crescente di paesi. Sulla base di questo presupposto, l'articolo cerca di comprendere se questa sempre più diffusa richiesta di un diritto di morire si fondi su motivazioni più complesse che affondano le loro radici nelle modificazioni delle forme del potere nelle società occidentali. Un potere sempre più rivolto alla regolazione delle vite delle persone trova infatti nella morte un ambito privo di interesse, e può pertanto agevolmente riconoscere un diritto di morire. Infine si domanda se questo nuovo diritto di autodeterminazione sia effettivamente in grado di incidere in modo sensibile sull'aspetto più crudele del morire nella società contemporanea che è rappresentato dalla solitudine del morente, relegato negli ospedali e allontanato dai luoghi degli affetti.
\end{abstract}

PALABRAS CLAVE: autodeterminazione, eutanasia, aiuto al suicidio, diritto alla salute, libertà.

ABSTRACT: The article analyzes the recent order of the Italian Constitutional Court, n. 207 of 2018, which opens up to the constitutional legitimacy, in certain circumstances, of aid to suicide, read as indicative of a general and recent trend aimed at giving acceptance to these forms of self-determination in an ever increasing number of countries. On the basis of this assumption, the article tries to understand if this increasingly widespread request for a right to die is based on more complex reasons that have their roots in the changes in the forms of power in Western societies. An increasingly power to regulate people's lives finds in death an area devoid of interest, and can therefore easily recognize a right to die. Finally, he wonders if this new right of self-determination is actually able to significantly affect the cruelest aspect of dying in contemporary society which is represented by the loneliness of the dying person, relegated to hospitals and removed from the places of affections.

KEYWORDS: self-determination, euthanasia, aid to suicide, right to health, freedom.

SUMARIO: SEZ. I. IL DIRITTO DI AUTODETERMINAZIONE TERAPEUTICA NELL'ORDINAMENTO ITALIANO. I. Il DIRITTO DI MORIRE COME MANIFESTAZIONE INDIVIDUALE TRA TRASFORMAZIONI DEL POTERE STATALE E RIFIUTO SOCIALE DELLA MORTE. II. L'AUTODETERMINAZIONE TERAPEUTICA NELLA GIURISPRUDENZA ITALIANA. III. L'ORDINANZA N. 204 DEL 2018 DELLA CORTE COSTITUZIONALE: VERSO UNA PIÙ PIENA AUTODETERMINAZIONE TERAPEUTICA. SEZ. II. IL DIRITTO DI MORIRE TRA MODIFICAZIONE DEL POTERE SOVRANO E RIFIUTO SOCIALE DELLE MORTE. IV. LE TRASFORMAZIONI DELLA SOVRANITÀ E IN DIRITTO DI MORIRE. V. LA MORTE DELLA FAMILIARITÀ DEI LUOGUI DOMESTICI. VI. IL TABÙ DELLA MORTE E IL DOVERE DI ESSERE FELICI. VII. LA SOLITUDINE DEL MORENTE TRA OSPEDALIZZAZIONE DELLA MORTE E POTERE MEDICO. 
SEZ. I. IL DIRITTO DI AUTODETERMINAZIONE TERAPEUTICA NELL'ORDINAMENTO ITALIANO

\section{IL DIRITTO DI MORIRE COME MANIFESTAZIONE DELL' AUTODETERMINAZIONE INDIVIDUALE TRA TRASFORMAZIONI DEL POTERE STATALE E RIFIUTO SOCIALE DELLA MORTE}

Il diritto di morire è un tema di grandissima attualità. Nella maggior parte degli Stati occidentali in cui non sono riconosciute forme di eutanasia attiva o di aiuto al suicidio, è molto accesa la discussione sul fondamento etico e giuridico di un diritto di autodeterminazione individuale che consenta al malato incurabile di essere aiutato a porre termine alla propria esistenza.

Il diritto di morire è un tema di grandissima attualità. Nella maggior parte degli Stati occidentali in cui non sono riconosciute forme di eutanasia attiva o di aiuto al suicidio, è molto accesa la discussione sul fondamento etico e giuridico di un diritto di autodeterminazione individuale che consenta al malato incurabile di essere aiutato a porre termine alla propria esistenza.

Se il diritto di rifiutare le cure, anche nel caso in cui dal rifiuto di cure derivi la morte, è oramai diventato un diritto consolidato nell'ambito degli ordinamenti liberaldemocratici, gli Stati che riconoscono la liceità di forme di eutanasia attiva volontaria o di pratiche di assistenza al suicidio sono ancora una minoranza.

Il diritto di morire, infatti, inteso come diritto del malato incurabile di decidere il momento in cui porre termine alla propria esistenza, non solo contraddice il principio di sacralità della vita, ma presenta degli indubbi tratti di contraddittorietà per cui è spesso difficile da concepire sul piano logico, prima ancora che con riferimento a parametri giuridici ed etici, proprio perché il suo esercizio, determinando la scomparsa della persona, comporta anche la perdita di ogni altro diritto ${ }^{1}$.

Tuttavia, proprio il generale riconoscimento del diritto di rifiutare le cure, interpretato come comprensivo del diritto di revocare un trattamento sanitario (salvavita) in atto, è un passaggio di carattere fondamentale che spinge nella direzione di una sempre più piena autodeterminazione del malato nelle fasi terminali della sua esistenza e ci offre argomenti giuridici, fondati soprattutto sul principio di eguaglianza, per sostenere la necessità di introdurre, per via legislativa, forme di tutela dei malati incurabili che richiedano di porre termine alla loro esistenza, da essi stessi considerata contrastante con la loro condizione esistenziale, con la loro personale idea di dignità e con il concetto che essi hanno di se stessi e della propria salute ${ }^{2}$.

\footnotetext{
${ }^{1}$ Cfr. A. D'Aloia, "Eutanasia (dir. cost.)”, in Digesto disc. pubbl., Torino 2012. Su questi temi cfr. C. Tripodina, Il diritto nell'età della tecnica. Il caso dell'eutanasia, Napoli, 2004.

${ }^{2}$ Sul concetto di salute cfr. P. Zatti, "Il diritto a scegliere la propria salute (in margine al caso S. Raffaele)", in Nuova giur. civ. comm., 2000, II, 1 ss. ove si legge: «Il diritto alla salute come diritto fondamentale esige un contenuto adeguato all'idea di persona come unità di tutti quegli aspetti che il pensiero, nel suo ragionare per distinzioni ed opposti, osserva e cataloga come "componenti" della persona; in particolare, corpo e psiche. il problema sta nella concatenazione di due aspetti evolutivi dell'idea di salute: a) quello per cui essa si espande agli aspetti psichici, o mentali, del benessere, e $b$ ) quello per cui il benessere si centra sulla soggettività, sull'esperienza che ciascuno ha del proprio stato fisico, mentale e -se di altri si tratta rispetto al mentale-spirituale» per cui «Salute diviene un concetto che esprime anzitutto una percezione di sé come soggetto integro».
} 
L'apertura dei sistemi giuridici verso una sempre maggiore affermazione dell'autodeterminazione terapeutica ${ }^{3}$ è indubbiamente in grado di ampliare le possibilità di scelta di chi è affetto da una malattia incurabile ed intende sottrarsi a una condizione fisica che, lungi dall'essere percepita come qualcosa assimilabile al concetto di vita o di salute, considera come una forma di violenza perpetrata sul suo corpo diventato una vera e propria prigione e fonte di sofferenze ${ }^{4}$.

In Italia, con l'ordinanza n. 207 del 2018 della Corte costituzionale, il diritto all'autodeterminazione terapeutica è stato interpretato come un diritto comprensivo non solo del diritto di rifiutare cure salvavita, ma anche della possibilità di determinare, in ben precise circostanze, il momento terminale della propria esistenza.

Questa ordinanza si inserisce in una tendenza verso il riconoscimento di un vero e proprio diritto di morire, testimoniata dal crescente numero di paesi in cui negli ultimi decenni è stato disciplinato e dal sempre più diffuso consenso che il principio di autodeterminazione trova nella società. Si pensi all'esito delle due consultazioni popolari sul death with dignity act dello stato dell'Oregon. Nel 1994 il favore verso questa legge fu del 51 per cento, mentre soli tre anni dopo i voti favorevoli oltrepassarono il 60 per cento. Del resto, la stessa Corte Suprema canadese quando, nel 2015, ha ammesso la legittimità costituzionale dell'aiuto al suicidio ${ }^{6}$ ha superato il suo precedente contrario del 1993 (sentenza Rodriguez v. British Columbia ${ }^{7}$, in cui aveva negato accoglimento ad una domanda simile) rilevando come nel corso degli ultimi anni fosse emersa una diversa interpretazione dei principi fondamentali che consentiva di superare il citato precedente.

Diventa pertanto interessante cercare di comprendere se questa sempre più diffusa richiesta di un diritto di morire si fondi su motivazioni più complesse che affondano le loro radici nelle modificazioni delle forme del potere nelle società occidentali. Un potere sempre più rivolto alla regolazione delle vite delle persone trova infatti nella morte un ambito privo di interesse $^{8}$, e può pertanto agevolmente riconoscere un diritto di morire.

Occorre infine domandarsi se questo nuovo diritto di autodeterminazione (in via di progressiva affermazione nelle società occidentali) sia effettivamente in grado di incidere in modo sensibile sulla condizione dei malati incurabili nella nostra società e sulla drammaticità dell'intero processo del morire che inizia con la malattia stessa. L'aspetto più crudele del morire nella società contemporanea è rappresentato dalla solitudine del morente, cioè dalla condizione di isolamento e di abbandono del malato terminale, conseguenza della crescente medicalizzazione della vita. Normalmente, infatti, il malato è sottratto al contesto familiare della propria abitazione, cioè il luogo in cui ha trascorso la vita e ha intessuto le relazioni affettive per

\footnotetext{
${ }^{3}$ Sul concetto di autodeterminazione terapeutica cfr. L. Chieffi, "Il diritto all'autodeterminazione del malato terminale: tecniche di interpretazione di un valore costituzionale", in Accademia Nazionale dei Lincei, Testamento biologico e libertà di coscienza, Roma, 2013, 137 e ss. e da ultimo Id., Il diritto all'autodeterminazione terapeutica. Origine ed evoluzione di un valore costituzionale, Giappichelli, Torino, 2019, passim.

${ }^{4}$ Sul punto cfr. G. Gemma, Mantenimento in vita di pazienti in stato vegetativo permanente: obbligo costituzionale di rispettare la loro volontà, in L. Foffani, C. Fregni, R. Lambertini, a cura di, Liber amicorum per Massimo Bione, Milano, Giuffré, 2011, p. 291 e ss.

${ }^{5} \mathrm{Si}$ ricorda che la Corte costituzionale ha elaborato alcuni principi che dovrebbero ispirare il legislatore nella redazione di una disciplina a questo proposito, ma non ha chiuso il caso. Ha infatti rinviato la decisione ad altra udienza, fissata nel settembre 2019, per dare al legislatore il tempo di approvare una nuova disciplina. Il caso, pertanto, è ben lontano dal potersi definire chiuso.

${ }^{6}$ Carter v. Canada (Attorney General), 2015 SCC 5.

${ }^{7}$ Rodriguez v. British Columbia (Attorney General), 3 SCR 519.

${ }^{8}$ FOUCAULT, M., "La volontà di sapere. Storia della sessualità", I [ed. or.: La volonté de savoir, Gallimard, Parigi, 1976], Feltrinelli, Roma, 2016.
} 
essere relegato negli ospedali, finendo così con l'essere sostanzialmente abbandonato a sé stesso e sottoposto unicamente alla distaccata vigilanza del potere medico. La morte viene nascosta negli ospedali perché rifiutata dalla nostra società individualista, tutta protesa alla incessante ricerca del benessere individuale, finendo per rappresentare un vero e proprio tabù e allontanata dai luoghi in cui si svolge la vita quotidiana.

Questa condizione di solitudine e di abbandono rappresenta forse l'aspetto più disumano della morte nelle attuali metropoli del mondo globalizzato, rispetto al quale il diritto di autodeterminazione (anche declinato nelle forme del diritto di morire) può fare ben poco se non è accompagnato da processo culturale che riattribuisca alla morte il suo ruolo (e il suo significato) nelle cose ordinarie della vita, invertendo quel processo che ci ha portato ad allontanarla dalla vista collettiva.

\section{L' AUTODETERMINAZIONE TERAPEUTICA NELLA GIURISPRUDENZA ITALIANA}

Il diritto all'autodeterminazione terapeutica è uno degli esempi paradigmatici in cui si percepisce in modo limpido come il numero e lo stesso contenuto dei diritti fondamentali, progressivamente, si adegui al modo in cui la società interpreta e sente i valori e i principi costituzionali ${ }^{9}$. Il riconoscimento di nuovi diritti fondamentali è, infatti, l'esito di una sintesi che compone un conflitto sociale e che si snoda normalmente attraverso un complesso e lungo processo che vede la partecipazione di una molteplicità di attori: dall'opinione pubblica ai giudici, dalla cultura, giuridica e non, al parlamento fino a coinvolgere associazioni, categorie professionali e gruppi di interesse. Siffatto processo è condizionato in maniera importante dal modo in cui la società interpreta i principi costituzionali, strutturalmente elastici ${ }^{10}$.

Le soluzioni giuridiche sul fine vita sono spesso giunte, infatti, a valle di un dibattito che ha coinvolto la società intera e la cultura giuridica in particolare; dibattito quasi sempre provocato da casi tragici che hanno scosso l'opinione pubblica. È stato così per l'affermazione del diritto di rifiutare le cure, ivi comprese quelle salvavita (e del diritto di revocare un precedente consenso dato a cure salvavita). Lo stesso percorso si sta ora riproponendo in questi ultimi tempi con riferimento all'ammissibilità di ipotesi di eutanasia attiva o di aiuto al suicidio: il caso del DJ Fabo Antoniani del 2017 in Italia come il recentissimo caso della signora María José Carrasco, in Spagna, ne sono un esempio.

Come accade nella maggior parte delle vicende bioetiche, anche in questo caso il ruolo della giurisprudenza si rivela essenziale. Nel passato sono stati proprio giudici a enucleare dai principi le regole da applicare a casi non espressamente disciplinati dalla legge, provocati dal progresso tecnologico che consentiva di tenere in vita pazienti che altrimenti avrebbero terminato la propria esistenza. Sulla giurisprudenza è ricaduto il compito di bilanciare i valori e i principi costituzionali. L'affermazione del right to refuse medical treatement ha trovato pertanto le sue basi teoriche nella giurisprudenza della Corte del New Jersey sul caso Quinlan - che impostò la vicenda nell'ambito del diritto alla privacy - ed in quella della Corte Suprema degli Usa sul caso Cruzan - che la ricondusse alla dottrina del consenso informato.

\footnotetext{
${ }^{9}$ LUCIANI, M., "L'interprete della Costituzione di fronte al rapporto fatto-valore. Il testo costituzionale nella sua dimensione diacrónica”, in Diritto e società, 2009, p. 20. V. M. Fioravanti, Le due trasformazioni costituzionali dell'età repubblicana, www.astrid-online, 200

${ }^{10}$ Cfr. VERONESI, P., "Rights on the move: come cambiano i diritti costituzionali (e le loro interpretazioni)", in Rivista di biodiritto (www.biodiritto.org), 2/2018.
} 
Anche la disciplina di ipotesi di eutanasia attiva o di forme di assistenza al suicidio è stata spesso tributaria dell'opera del giudice. Basti citare, a titolo esemplificativo: la derivazione giurisprudenziale della legislazione olandese in materia di eutanasia; la sentenza della Corte Suprema canadese del 2015 che ha annullato l'incriminazione dell'assistenza al suicidio e, non ultima, la stessa ordinanza della Corte costituzionale italiana in materia di fine vita dell'ottobre 2018, di cui si parlerà oltre ${ }^{11}$.

Fino alla citata decisione della Corte Costituzionale (i cui esiti, come si chiarirà, sono ancora aperti), il diritto di autodeterminazione terapeutica del malato si è affermato, per via giurisprudenziale, come diritto di rifiutare le cure e diritto al consenso informato ${ }^{12}$. Diritti ancorati, da un lato, sulla libertà individuale (garantita dall'art. 13 Cost.) e, dall'altro, sull'art. 32, II comma della Costituzione il quale, imponendo una legge perché un determinato trattamento sanitario assuma il carattere dell'obbligatorietà, fonda, secondo la lettura corrente, il più ampio diritto individuale del rifiuto di trattamenti sanitari ${ }^{13}$. Le risultanze della giurisprudenza in materia sono state successivamente formalizzate e razionalizzate nell'ambito della legge n. 219 del 2017 in materia di dichiarazioni anticipate di trattamento ${ }^{14}$.

Il riconoscimento di un siffatto diritto di autodeterminazione del malato è l'esito di un lungo processo giurisprudenziale cui si è giunti a piccoli passi ed in cui ogni intervento giurisprudenziale ha posto un piccolo ma essenziale mattone per arrivare alla costruzione (dell'ancora incompleto, a parere di chi scrive) statuto della libertà di disporre del proprio corpo con riferimento alle decisioni in merito alla propria salute.

Era il 16 dicembre 2006 quando il tribunale di Roma rifiutò di riconoscere a Piergiorgio Welby, malato di SLA e tenuto in vita attraverso un respiratore artificiale, il diritto di autodeterminarsi con riferimento alla propria salute, negando l'autorizzazione al medico che avrebbe dovuto staccare il respiratore artificiale. Il giudice capitolino, infatti, sebbene avesse ricostruito la sussistenza di un ampio diritto di autodeterminazione individuale derivante dagli articoli 13 e 32 della Costituzione repubblicana, osservò che l'ordinamento giuridico dovesse essere considerato complessivamente, per cui «allorché la richiesta riguardi il rifiuto o l'interruzione di trattamenti medici di mantenimento in vita del paziente, il principio di fondo ispiratore è quello della indisponibilità del bene vita», sicché, nonostante la ricostruzione del quadro costituzionale, tale diritto di autodeterminazione doveva considerarsi «non concretamente tutelato dall'ordinamento» ${ }^{15}$.

Esattamente un anno dopo la Corte di cassazione ${ }^{16}$ realizzava un importante passo avanti nel percorso verso l'affermazione del diritto di autodeterminazione terapeutica, modificando in modo sensibile l'interpretazione del diritto positivo interno in materia di fine vita. Nella citata sentenza, infatti, il giudice della legittimità ha consentito ad una donna, Eluana Englaro (da anni in stato vegetativo permanente e alimentata e idratata tramite un sondino naso

\footnotetext{
${ }^{11}$ Si tratta dell'ordinanza n. 207 del 2018 della Corte costituzionale sul caso del DJ Fabo Antoniani con la quale è stata messa in discussione la legittimità costituzionale del divieto generalizzato di aiuto al suicidio.

${ }^{12}$ Cfr., in particolare, Cass. civ., sez. I, sent. 16 ottobre 2007, n. 21748 (che si legge su www.biodiritto.org)

${ }^{13}$ Cfr. Corte costituzionale, sent. n. 438 del 2008

${ }^{14} \mathrm{Su}$ questi temi la letteratura è estesissima. Cfr. da ultimo CHIEFFI, L., Il diritto all'autodeterminazione terapeutica. Origine ed evoluzione di un valore costituzionale, Torino, Giappichelli, 2019, passim.

15 Tribunale di Roma, ordinanza del 16 dicembre 2006. Il testo dell'ordinanza è reperibile in https://www.eius.it/giurisprudenza/2006/150

${ }^{16}$ Cfr. la già citata Cass. civ., sez. I, sent. 16 ottobre 2007, n. 21748.
} 
gastrico), di ottenere il distacco dei trattamenti di idratazione e alimentazione sulla base del principio del consenso informato ${ }^{17}$.

La sentenza Englaro si fonda su una lettura del diritto alla salute che, sebbene fosse stata spesso affermata in dottrina e in giurisprudenza, aveva avuto difficoltà a trovare applicazione proprio con riferimento ai casi di fine vita, apparendo contraddittorio invocare una disposizione che tutela la salute come diritto fondamentale dell'individuo, per assumerlo a caposaldo del diritto di lasciarsi morire.

Nella sentenza Englaro la Cassazione ha chiarito, da un alto, che «il consenso afferisce alla libertà morale del soggetto e alla sua autodeterminazione»e, dall'altro, che un trattamento sanitario obbligatorio ha precisi limiti tracciati dall'art. 32 Cost. al di fuori dei quali la salute, «come tutti i diritti di libertà, implica la tutela del suo risvolto negativo: il diritto di perdere la salute, di ammalarsi, di non curarsi, di vivere le fasi finali della propria esistenza secondo canoni di dignità umana propri dell'interessato, finanche di lasciarsi morire».

Proprio questa ampia concezione della salute come diritto di libertà si è consolidata ed ha consentito di risolvere altre questioni di fine vita. Il Tribunale di Cagliari, nel 2016, ha autorizzato i medici a staccare il respiratore artificiale ad un malato di SLA che ne aveva fatto richiesta perché il diritto all'autodeterminazione terapeutica, «come tutti i diritti di libertà, è evidentemente condizionato dalle convinzioni etiche proprie del titolare del diritto medesimo ed è altresì coerente con la nuova dimensione che oggi ha assunto la salute, non più intesa come semplice assenza di malattia, ma come stato di benessere fisico e psichico, coinvolgente, in relazione alla percezione che ciascuno ha di sé, anche gli aspetti interiori della vita e le relazioni con gli altri» ${ }^{18}$.

Che il principio di autodeterminazione terapeutica contempli il diritto di lasciarsi morire (anche nei casi in cui questo diritto si realizza necessariamente attraverso un'attività positiva di un terzo) è pertanto oramai comunemente accettato ed è stato recentemente affermato dallo stesso legislatore, con la legge n. 219 del 2017, che ha disciplinato per la prima volta nell'ordinamento italiano le dichiarazioni anticipate di trattamento. Tale legge ha chiarito che il diritto di autodeterminazione terapeutica ricomprende non solo il diritto di lasciarsi morire, rifiutando (pure con disposizioni anticipate) trattamenti salvavita, ma anche quello di chiedere la sedazione palliativa profonda continua in caso di «prognosi infausta a breve termine o di imminenza di morte».

\footnotetext{
${ }^{17}$ In particolare, poiché non esistevano dichiarazioni anticipate di trattamento (che peraltro la legge al tempo non contemplava), la Corte di cassazione ricostruì la volontà della donna attraverso un processo complesso che, da un lato, ricorda il substitute judgment della giurisprudenza del New Jersey risalente al caso Quinlan (fondandosi, almeno in parte, sulla ricostruzione della personalità dell'incapace attraverso la testimonianza dei suoi cari e degli amici più stretti), dall'altro non ha mancato di effettuare (o perlomeno di tentare) una ricerca di prove (per quanto possibile), raccogliendo testimonianze di amici che ricordavano come Eluana avesse più volte reagito di fronte a casi come era quello in versava in quel momento, affermando che mai avrebbe accettato una simile condizione di vita. Una volta accertata processualmente la («presunta») volontà di Eluana, la decisione della Cassazione si è giuridicamente fondata sulla mancanza del consenso alla prosecuzione dell'idratazione e della nutrizione artificiali.

${ }^{18}$ Tribunale di Cagliari, decreto del 16 luglio 2016. Su tale decisione cfr. D'ALOIA, A., "Il caso Piludu e il diritto di rifiutare le cure (anche life-sustaining)", in Studium Iuris, 2018, p. 1462 ss.
} 


\section{L'ORDINANZA N. 204 DEL 2018 DELLA CORTE COSTITUZIONALE: VERSO UNA PIÙ PIENA AUTODETERMINAZIONE TERAPEUTICA}

Mentre si discuteva dell'approvazione della legge n. 219 del 2017, la realtà portava all'attenzione della giurisprudenza un caso capace di mettere a dura prova il quadro normativo appena formalizzato dal legislatore.

Il DJ Fabo Antoniani era stato vittima, nel 2104, di un incidente automobilistico che, sebbene lo avesse lasciato nella pienezza delle sue facoltà mentali, gli aveva provocato gravi conseguenze (come la cecità e la paralisi totale), con impossibilità di alimentarsi autonomamente e con la necessità di essere aiutato a respirare, sebbene in modo non continuativo. Per uno strano scherzo del destino, inoltre, a questa totale paralisi non si era accompagna - come spesso accade - la perdita della sensibilità, per cui, sempre come effetto dell'incidente, era stato colpito da una sindrome dolorosa che si manifestava con spasmi ricorrenti tra le 20 e le 60 volte al giorno ${ }^{19}$, che potevano raggiungere un'intensità insopportabile $^{20}$. Dopo infruttuosi tentativi di cura, anche sperimentali, Fabo Antoniani maturò la decisione di porre fine alla propria esistenza, ottenendo in Svizzera quell'assistenza al suicidio assolutamente vietata dalla legislazione italiana. Il signor Marco Cappato, che lo aveva accompagnato in auto in Svizzera alla Clinica Dignitas, è stato così indagato per il reato di istigazione al suicidio previsto dall'art. 580 c.p.

Nell'ambito del processo penale contro Marco Cappato, la Corte d'Assise di Milano ${ }^{21}$ ha sollevato questione di legittimità costituzionale con riferimento al delitto di istigazione al suicidio nella parte in cui «incrimina le condotte di aiuto al suicidio in alternativa a quelle di istigazione e, quindi, a prescindere dal loro contributo alla determinazione o al rafforzamento del proposito di suicidio».

Nell'ordinanza di rimessione alla Consulta, la Corte d'Assise ha ricordato che il bene giuridico originariamente protetto dall'art. 580 era la pretesa dello Stato sulle vite individuali. Il codice penale e l'art 5 del codice civile, contenente il divieto di atti di disposizione del proprio corpo limitativi dell'integrità fisica, sancivano il principio di indisponibilità del bene «vita». Le persone erano considerate in funzione del loro apporto agli interessi della collettività ${ }^{22}$, di modo che la stessa salute non integrava affatto un diritto ma, al contrario, un dovere (quello cioè di stare in salute) che l'individuo aveva verso la società.

L'assoluta indisponibilità del bene vita determinava, pertanto, che il suicidio fosse considerato un atto antigiuridico, sebbene non punito esclusivamente per ragioni di politica criminale. La non punibilità del suicida era dovuta ad una ragione di carattere preventivo, nel senso che una eventuale sanzione penale contro il mancato suicida avrebbe spinto l'aspirante suicida a trovare strade più efficaci per la realizzazione del proposito, frustrando così la ragione stessa della previsione della sanzione penale. Logico corollario di questa impostazione era l'antigiuridicità non solo dell'istigazione ma anche del mero aiuto al suicidio, in quanto in entrambi i casi si integrava una cooperazione in un atto antigiuridico (il suicidio) che non era punito solo per le dette ragioni di opportunità.

\footnotetext{
${ }^{19}$ Come riportato nella richiesta di archiviazione formulata dalla procura in data 2 maggio 2017 pubblicata su dirittopenalecontemporaneo.it

${ }^{20}$ Come testimoniato dal medico consulente della procura.

${ }^{21}$ Corte d'Assise di Milano, ord. 14 febbraio 2018. L'ordinanza si legge in www.biodiritto.org

${ }^{22}$ Ad esempio, il reato di aborto, previsto nel codice Rocco del 1930, era classificato come un delitto contro la integrità e la sanità della stirpe: impedire a una donna di procreare non era considerato un attentato contro l'interesse della donna o della vita in fieri, ma un attentato nei confronti degli interessi statali alla crescita demografica.
} 
La Costituzione repubblicana ha sovvertito questo quadro valoriale. Il principio personalistico non solo valorizza l'autonomia individuale, ma impedisce di strumentalizzare l'uomo per finalità collettive, sancendo, nella ricostruzione della Corte di Assise, il pieno riconoscimento della libertà di autodeterminazione.

La Corte d'Assise, pertanto, ha sottoposto alla Corte costituzionale la questione di legittimità costituzionale dell'incriminazione dell'art. 580 nella parte in cui sanziona la condotta di aiuto al suicidio che non abbia influito sul processo volitivo del suicida ${ }^{23}$. Se la sfera di autodeterminazione della persona capace è così ampia da far ritenere che essa sia «comprensiva della libertà di scegliere come e quando morire ${ }^{24}$, anche il suicidio della persona capace rientra in tale sfera di libertà. Ne consegue che l'incriminazione di un comportamento che sia meramente agevolativo della decisione presa liberamente, da persona capace, non può essere considerato illecito.

È interessante osservare che la Corte di Assise di Milano non menziona l'art. 32 Cost. fra i parametri costituzionali violati. Questa omissione probabilmente trae la sua ragion d'essere dalla circostanza che il diritto di autodeterminazione terapeutica, per come si è consolidato nel corso degli anni, rende complesso richiamare l'art. 32 della Costituzione per giustificare l'istanza di governo delle fasi finali della propria esistenza al di fuori della ipotesi del rifiuto di cure. La dottrina e la giurisprudenza, infatti, hanno utilizzato questa disposizione soprattutto con riferimento alla regola dettata nel II comma, quella cioè che vieta i trattamenti sanitari obbligatori.

Pertanto, l'idea che si è consolidata, prima nella giurisprudenza e nella dottrina, ed è poi stata accolta dalla legge n. 219 del 2017 sul cosiddetto testamento biologico, è che il diritto di autodeterminazione del malato, che si fonda sul II comma dell'art. 32, si declina nel senso di attribuire al malato il diritto di rifiutare i trattamenti sanitari e il diritto al consenso informato.

Questa interpretazione determina che, ove non vi sia un trattamento sanitario in corso, una richiesta di autodeterminazione con riferimento al momento finale della propria esistenza non possa fondarsi sul secondo comma dell'art. 32 della Costituzione ${ }^{25}$.

La motivazione della Corte di appello di Milano si inserisce chiaramente in questo filone interpretativo ${ }^{26}$.

${ }^{23}$ BISSARO, S., Le (non insuperabili) strettoie del giudizio incidentale alla prova del caso "Cappato". Riflessioni a margine della questione di legittimità costituzionale dell'art. 580 c.p. promossa dalla Corte d'Assise di Milano, forumcostituzionale, 2018, 4. D'AMICO. M., "Sulla (il)legittimità costituzionale della norma penale che incrimina l'istigazione al suicidio: alcune considerazioni critiche a margine del caso Cappato", in www.giurisprudenzapenale.com, 2017.

${ }^{24}$ Corte d'Assise di Milano, ord. 14 febbraio 2018.

${ }^{25}$ Come è stato osservato da GIANELLO, S., La strada impervia del giudizio incidentale. Nota all'ordinanza di rimessione nel «processo Cappato», in www.diritticomparati.it, 26 febbraio 2018: « La via astrattamente più corretta, seppur non priva di insidie, sarebbe consistita nella proposizione di una "chirurgica" richiesta additiva alla Corte che avrebbe potuto dichiarare l'illegittimità dell'art. 580 c.p. nella parte in cui non prevede la non punibilità di chi agevola il suicidio alla presenza di una precisa situazione di fatto data dal sommarsi di elementi quali l'assoluta sofferenza del malato, la sua impossibilità di procedere ex se nonostante percepisca la sua esistenza come non più dignitosa, lo stato di una certificazione medica che attesti l'irreversibilità della sua patologia, etc.». Questo perché «La richiesta tout court di ablazione della norma nella parte in cui sanziona l'agevolazione al suicidio, in assenza di limiti e ragioni concrete idonee a giustificarne l'accoglimento sul lato delle condizioni di salute divenute insostenibili per il malato può difficilmente dirsi figlia di un imperativo di ordine costituzionale» (Ibidem).

${ }^{26}$ Sull'ordinanza di rimessione alla Corte cfr. ZAGREBELKY, V., Autodeterminazione, suicidio assistito e Convenzione europea dei diritti umani e delle libertà fondamentali, Relazione al convegno "Autodeterminazione terapeutica e questioni di fine vita" (Roma, 23 ottobre 2017), consultabile sul sito associazionelucacoscioni.it.; D'AMICO, M., "Sulla (il)legittimità costituzionale della norma penale che incrimina l'istigazione al suicidio: alcune 
In altri termini, la Corte di Assise sarebbe stata in un certo senso "costretta" a fare a meno dell'art. 32 Cost. perché nella fattispecie sottoposta al suo esame non veniva in rilievo un rifiuto di cure ma la richiesta di un'azione positiva causativa della morte. Infatti, come è stato osservato, «facendo leva sul principio personalistico enunciato dall'art. 2 Cost. e su quello dell'inviolabilità della libertà individuale consacrato nell'art. 13, comma 1 , Cost., la Corte milanese cerca di affrancare il «diritto di autodeterminarsi anche in ordine alla fine della propria esistenza» dai confini del rapporto terapeutico in cui le note sentenze pronunciate nelle vicende Welby ed Englaro lo avevano inserito ${ }^{27}$.

La Corte costituzionale ha dato alla questione una risposta che al momento è solo provvisoria, emanando un'ordinanza del tutto atipica, definita da alcuni di «incostituzionalità differita $»^{28}$. In particolare, la Corte ha chiarito che l'incriminazione tout court dell'aiuto a suicidio è incostituzionale, ma non ha dichiarato l'annullamento dell'art. 580 c.p. Con detta ordinanza la Corte ha fissato alcuni principi cui il legislatore si dovrebbe attenere nel dettare la nuova disciplina e si è aggiornata al 24 settembre 2019, di modo che, nel caso di inerzia del legislatore, potrà eventualmente dichiarare l'incostituzionalità dell'art. 580 c.p.

Per quanto attiene agli aspetti di merito, la Consulta ha respinto la ricostruzione della Corte di Assise affermando che «l'incriminazione dell'aiuto al suicidio non può essere ritenuta incompatibile con la Costituzione» ${ }^{29}$ in virtù «di una concezione astratta dell'autonomia individuale ${ }^{30} \mathrm{e}$ indipendentemente dalla considerazione delle condizioni personali di chi chiede di essere aiutato a morire. Tuttavia, il giudice delle leggi ha sviluppato un percorso argomentativo per venire incontro all'istanza fondamentale posta dall'ordinanza, quella cioè di non considerare illecito il comportamento di Marco Cappato.

La Corte ha ricondotto il caso nell'ambito dell'art. 32 della Costituzione, cioè del diritto di autodeterminazione terapeutica senza distaccarsi sensibilmente dall'interpretazione che di tale diritto si è consolidata nella giurisprudenza che lo ha declinato essenzialmente come diritto al rifiuto di cure. Nell'economia dell'ordinanza della Corte Costituzionale assume, infatti, un ruolo centrale la circostanza che Fabo Antoniani avrebbe potuto fare uso del diritto già previsto dall'ordinamento positivo vigente, cioè quello di porre termine alla propria esistenza attraverso il rifiuto di cure salvavita (come il respiratore), alle quali, sebbene non continuativamente, era sottoposto.

Ha osservato a tale proposito la Corte Costituzionale che «nel caso oggetto del giudizio a quo l'interessato richiese l'assistenza al suicidio, scartando la soluzione dell'interruzione dei trattamenti di sostegno vitale con contestuale sottoposizione a sedazione profonda (soluzione che pure gli era stata prospettata), proprio perché quest'ultima non gli avrebbe assicurato una morte rapida. Non essendo egli, infatti, totalmente dipendente dal respiratore artificiale, la morte

\footnotetext{
considerazioni critiche a margine del caso Cappato", in Giurisprudenza Penale Web, 2017; CASONATO, C., "Fine vita: diritto all'autodeterminazione", in Il Mulino, n. 4/17, p. 597 ss.

${ }^{27}$ BATTAGLIA, G., "La questione di legittimità costituzionale dell'art. 580 c.p.: una tappa (necessaria?) del caso Cappato", in Quaderni costituzionali, 2/2018, 493 e ss. La citazione è a pag. 494.

${ }^{28}$ BIGNAMI, M., Il caso Cappato alla Corte costituzionale: un'ordinanza ad incostituzionalità differita, in Quest. giust. (19 novembre 2018). Sull'ordinanza cfr.: in senso critico, RUGGERI, A., Pilato alla Consulta: decide di non decidere, perlomeno per ora (a margine di un comunicato sul caso Cappato), in www.giurcost.org 2018, fasc. 3; in senso adesivo, ADAMO, U., La Corte è "attendista"... «facendo leva sui propri poteri di gestione del processo costituzionale». Nota a Corte cost. n. 207 del 2018, in www.forumcostituzionale.it; da ultimo, VERONESI, P., Un'incostituzionalità (solo) "di fatto" del reato di aiuto al suicidio: in attesa del seguito del "caso Cappato", in Studium Iuris, 2019.

${ }^{29}$ Corte Cost, ord. 207/2018, punto 8 del considerato in diritto.

${ }^{30}$ Corte Cost, ord. 207/2018, punto 7 del considerato in diritto.
} 
sarebbe sopravvenuta solo dopo un periodo di apprezzabile durata, quantificabile in alcuni giorni: modalità di porre fine alla propria esistenza che egli reputava non dignitosa e che i propri cari avrebbero dovuto condividere sul piano emotivo». La Corte ha argomentato pertanto in termini di ragionevolezza: «Se, infatti, il cardinale rilievo del valore della vita non esclude l'obbligo di rispettare la decisione del malato di porre fine alla propria esistenza tramite l'interruzione dei trattamenti sanitari - anche quando ciò richieda una condotta attiva, almeno sul piano naturalistico, da parte di terzi (quale il distacco o lo spegnimento di un macchinario, accompagnato dalla somministrazione di una sedazione profonda continua e di una terapia del dolore) - non vi è ragione per la quale il medesimo valore debba tradursi in un ostacolo assoluto, penalmente presidiato, all'accoglimento della richiesta del malato di un aiuto che valga a sottrarlo al decorso più lento - apprezzato come contrario alla propria idea di morte dignitosa - conseguente all'anzidetta interruzione dei presidi di sostegno vitale».

Per cui, conclude il giudice delle leggi, « Entro lo specifico ambito considerato, il divieto assoluto di aiuto al suicidio finisce, quindi, per limitare la libertà di autodeterminazione del malato nella scelta delle terapie, comprese quelle finalizzate a liberarlo dalle sofferenze, scaturente dagli artt. 2, 13 e 32, secondo comma, Cost., imponendogli in ultima analisi un'unica modalità per congedarsi dalla vita, senza che tale limitazione possa ritenersi preordinata alla tutela di altro interesse costituzionalmente apprezzabile, con conseguente lesione del principio della dignità umana, oltre che dei principi di ragionevolezza e di uguaglianza in rapporto alle diverse condizioni soggettive (art. 3 Cost.: parametro, quest'ultimo, peraltro non evocato dal giudice a quo in rapporto alla questione principale, ma comunque sia rilevante quale fondamento della tutela della dignità umana)».

Così argomentando, dunque, la Corte non si è allontanata dalla consolidata lettura che interpreta il diritto di autodeterminazione terapeutica strettamente legato al II comma dell'art. 32.

La Corte infatti non ha considerato che la richiesta di Fabo Antoniani di porre termine alla propria esistenza potesse avere un fondamento autonomo rispetto al rifiuto di cure. Al contrario, il giudice delle eleggi in tanto ha effettuato un'apertura verso una forma di assistenza al suicidio in quanto, nel caso di specie, tale assistenza si manifestava come una modalità alternativa a un diritto già chiaramente sancito: quello di rifiutare le cure ${ }^{31}$. Il diritto di autodeterminazione terapeutica, pertanto, nel ragionamento della Consulta, continua a fondarsi sul secondo comma dell'art. 32, cioè sul diritto al rifiuto di cure; l'apertura a ipotesi ai assistenza al suicidio non ha indotto la Consulta a fondare queste forme di autodeterminazione direttamente sullo statuto di libertà connesso al diritto alla salute, considerato come comprensivo anche del suo aspetto negativo (aspetto negativo della libertà che potrebbe essere in grado di attribuire alla persona malata, in alcune determinate circostanze, di determinare il momento della fine della propria esistenza) ${ }^{32}$.

\footnotetext{
${ }^{31}$ Sul punto cfr. CASONATO, C., "I limiti all'autodeterminazione individuale al termine dell'esistenza: profili critici", in Dir. Pubbl. Eur. e Comp., 1/2018 che individua la soluzione in una rinnovata lettura del consenso informato. Osserva 1'Autore: «Se tale linea evolutiva del principio del consenso può essere condivisa, pare essere giunto il momento di superarne la formula originaria, stretta all'interno di logiche corporali troppo anguste e ormai desuete, per considerare la tutela di una più ampia autodeterminazione individuale, inclusiva dei profili non solo fisici ma complessivamente esistenziali e ontologici della persona in tutte le fasi della sua vita. Il principio del con- senso ecco la strada qui proposta per uscire dall'impasse sopra indicata - dovrebbe emanciparsi dal mero originario riferimento ad un intervento medico sul corpo, e trovare un più ampio e complessivo riferimento nel rispetto e nella promozione delle scelte sull'esistenza, che, in fasi particolari e delicate come quelle del fine vita, si saldano strettamente con la biografia, con l'identità e la struttura morale della persona malata » (ivi, 18).

32 Sul punto cfr. MORANA, D., "A proposito del fondamento costituzionale per il "consenso informato» ai trattamenti sanitari: considerazioni a margine della sent. n. 438 del 2008”, in Giur. Cost., 2008, 4970 e ss.
} 
Eppure una simile interpretazione sarebbe stata possibile alla luce della Carta costituzionale e dell'evoluzione interpretativa che ha avuto il concetto di salute nella dottrina e nella giurisprudenza.

La Carta costituzionale, infatti, in modo assolutamente innovativo per il tempo in cui fu approvata, ha inquadrato la salute come una libertà «fondamentale» dell'individuo, in piena coerenza con il fondamento personalistico su cui si fonda la Costituzione repubblicana che valorizza gli ambiti di autonomia individuale. Per cui è su questo solenne e chiaro riconoscimento della libertà di salute che si potrebbe fondare il diritto di autodeterminazione terapeutica senza doverlo necessariamente legare al secondo comma dell'art. 32 il quale, se ben si riflette, pone una regola che ha la funzione di rafforzare il principio del primo comma. Regola che è peraltro una necessaria applicazione dello statuto di libertà in cui la Costituzione ha ricondotto la salute. Il diritto di rifiutare i trattamenti sanitari, così come il principio del consenso informato per ogni intervento terapeutico, si fondano sulla declinazione in termini di libertà fondamentale della salute. Il senso del secondo comma, pertanto, non è quello di fondare il diritto di autodeterminazione terapeutica (già contenuto nel primo) e nemmeno quello di stabilire la regola della volontarietà dei trattamenti sanitari (anch'essa già desumibile dal primo comma).

Il II comma dell'art. 32 assume un'importanza centrale con riferimento alle limitazioni di tale libertà in ambito terapeutico. Tale disposizione chiarisce, infatti, entro quali limiti possa essere coartata la libertà di salute, prevedendo che tale compressione (cioè un trattamento sanitario obbligatorio), non solo debba avvenire con legge, ma che tale legge non è libera nel contenuto perché non può oltrepassare i limiti connessi «al rispetto della persona umana».

Questo significa che la strada per limitare la libertà di salute è molto stretta.

Innanzitutto, non un generico interesse pubblico può consentire tale limitazione, ma solo esigenze volte alla tutela della salute collettiva. Tuttavia, il rispetto della persona umana invocato dalla Costituzione al secondo comma dell'art. 32 esige che una limitazione della sfera di autodeterminazione terapeutica in tanto è legittima in quanto sia volta anche alla tutela della salute individuale della persona obbligata. Il caso paradigmatico è quello della vaccinazione obbligatoria, in cui viene in rilievo non solo la tutela della collettività (dal rischio di contagio) ma anche quella della salute individuale. Vi deve cioè essere un doppio contestuale beneficio (per la collettività e per l'individuo). Al di fuori di questa ipotesi, la libertà di autodeterminazione terapeutica riprende il suo pieno statuto, quello cioè dell'assoluta incoercibilità.

Il contenuto pregnante del secondo comma non è pertanto la regola che prescrive che «nessun trattamento obbligatorio può essere imposto se non in base a una legge», ma la parte che segue, cioè il contro limite che tutela la persona umana contro le limitazioni della sua autodeterminazione terapeutica, cioè il principio del «rispetto della persona umana». Questo contro limite integra un principio che è in grado di rafforzare l'ambito di autodeterminazione terapeutica, limitando chiaramente la legittimità di eventuali compressioni e sancendo così un divieto assoluto di strumentalizzazione della persona per finalità ultra individuali.

La lettura congiunta del primo comma dell'art. 32, che qualifica la salute come un «fondamentale diritto dell'individuo»e il contro limite di cui al secondo comma, cioè «il rispetto della persona umana», sono in grado di fare della libertà di autodeterminazione terapeutica una libertà tutelata in modo rafforzato, in quanto essa è assolutamente incoercibile al di fuori delle legittime ipotesi di trattamenti sanitari obbligatori. 
Se la ricostruzione proposta è fondata, allora sia il diritto al rifiuto di cure che il diritto al consenso informato si fondano proprio sullo statuto di libertà connessa alla salute, cioè sul primo comma dell'art. 32. In maniera non diversa si dovrebbe ragionare anche con riferimento al diritto di determinare il momento in cui porre fine alla propria morte in quei casi di malattia grave e incurabile indipendentemente dalla circostanza che sussista o meno un trattamento salvavita in atto.

Se la salute, intesa come libertà, è comprensiva anche del suo aspetto negativo, come chiaramente affermato dalla giurisprudenza ${ }^{33}$, se non si può ritenere «che il diritto alla autodeterminazione terapeutica del paziente incontri un limite allorché da esso consegua il sacrificio del bene della vita ${ }^{34}$, allora si deve riconoscere che il diritto alla salute comprende non solo il diritto di rifiutare le cure salvavita e di revocare il consenso a quelle in corso, ma anche il diritto di determinare il momento finale della propria esistenza in tutti quei casi in cui la malattia sia talmente devastante che contrasti con l'idea di dignità della persona e con il modo in cui percepisce se stessa e il proprio corpo. L'ordinanza 207 della Corte costituzionale ha riconosciuto chiaramente che la richiesta di porre fine alla propria esistenza possa avere una finalità terapeutica laddove afferma «la libertà di autodeterminazione del malato nella scelta delle terapie, comprese quelle finalizzate a liberarlo dalle sofferenze». Con un'unica, ma importante precisazione. Mentre il diritto al rifiuto di cure è chiaramente operativo ex se, quello di determinare il momento finale della propria esistenza ha invece necessario bisogno dell'opera di precisazione del legislatore che ne deve stabilire non solo i confini con riferimento a oggettive condizioni di salute che ne consentano l'esercizio, ma anche le persone facoltizzate ad intervenire per esaudire la richiesta e le relative modalità di attuazione.

Il caso di Fabo Anoniani ha avuto pertanto il merito di far emergere chiaramente l'angustia dei risultati cui era giunto il diritto positivo vigente in materia ${ }^{35}$. Il caso di specie ci ha infatti messi di fronte a una contraddizione tra due opzioni per la realizzazione del medesimo scopo, entrambe richiedenti una condotta di terzi: da un lato, quella che la Corte di appello di Milano definisce come «la strada italiana», cioè l'interruzione di qualsiasi trattamento accompagnato dalla sedazione profonda, dall'altro quella che la stessa Corte di appello individua come «la strada svizzera». Strade alternative per ottenere lo stesso risultato, con la paradossale conseguenza che una rappresenta estrinsecazione di un diritto costituzionalmente tutelato, mentre l'altra concreta un'ipotesi di reato.

Da questa contraddizione, tuttavia, se ne esce solamente superando la consolidata interpretazione che fonda l'autodeterminazione terapeutica sul divieto di trattamenti sanitari obbligatori e non sullo statuto di libertà con cui la nostra Costituzione dà corpo al fondamentale diritto alla salute.

\footnotetext{
${ }^{33}$ Cfr., tra le altre, Cass., sez. civ., sent. n. 21748/2007 e Tribunale di Cagliari, decreto del 16 luglio 2016.

${ }^{34}$ Così Cass., sez. civ., sent. n. 21748/2007, punto 6.1 delle Motivazioni in diritto.

${ }^{35}$ Sul punto cfr. DONINI, M., "La necessità di diritti infelici. Il diritto di morire come limite all'intervento penale", in Riv. It. Med. Leg., fasc. 2/2016. P. 555 ss.
} 


\section{SEZ. II. IL DIRITTO DI MORIRE TRA MODIFICAZIONE DEL POTERE SOVRANO E RIFIUTO SOCIALE DELLE MORTE}

\section{LE TRASFORMAZIONI DELLA SOVRANITÀ E IL DIRITTO DI MORIRE}

La vicenda italiana ci mostra come anche i Paesi culturalmente più refrattari si stiano avviando ad ammettere il diritto di morire. Una trasformazione profonda se si pensa che ancora tra la fine del settecento e l'inizio dell'ottocento il suicidio era considerato un illecito gravissimo perché il suicida si arrogava un diritto di cui potevano disporre solo Dio o lo Stato ed era pertanto punito con l'oltraggio del cadavere e con la confisca dei beni del defunto. Una punizione pubblica e crudele per dare il senso che il potere statale riacquistava il suo pieno dominio su tutto ciò che restava del defunto: il suo corpo e i suoi beni. Una punizione che doveva fungere da monito alla collettività affinché ricordasse che solo il potere statale (o quello divino) potessero disporre della morte.

L'apertura degli ordinamenti giuridici contemporanei a forme sempre più ampie di autodeterminazione comprensive del diritto di morire rappresenta un indice circa la trasformazione delle forme e delle strutture del potere.

La sovranità statuale è infatti una categoria in crisi: al potere sovrano di un tempo si è progressivamente sostituito un potere meno visibile ma più efficace che si manifesta in meccanismi complessi e reticolari volti alla regolamentazione della vita; al concetto giuridico di goverment si è lentamente sostituito quello sociologico di governance. Per dirla con Michel Foucault, «al vecchio diritto di far morire o di lasciar vivere si è sostituito un potere di far vivere o di respingere nella morte» per cui è «ora sulla vita e lungo tutto il suo svolgimento che il potere stabilisce la sua presa» mentre «la morte ne è il limite, il momento che lo distrugge; diventa il punto più segreto dell'esistenza, il più privato» ${ }^{36}$.

Se il potere del sovrano era volto al governo di un determinato territorio e si fondava sulla morte (la sovranità è infatti una nozione romanistica che trova il proprio fondamento nel diritto romano ${ }^{37}$ in cui il pater familias non solo aveva un potere assoluto sui suoi beni, ma poteva persino disporre della vita dei membri della famiglia), il potere attuale non è più centralizzato, è rivolto al governo delle vite e trova pertanto nella morte il suo più chiaro limite. Per questo se ne disinteressa. Se il potere è rivolto alla vita, la morte ne segna il confine.

Foucault ha studiato la trasformazione del potere nell'età che egli stesso definisce biopolitica, cioè nell'età in cui la crescita del sistema produttivo di stampo capitalistico si è strettamente legata non più al governo degli stati intesi come enti territoriali, ma al governo dei più disparati aspetti che riguardano la vita biologica della popolazione (dalla salute, alla cura dei corpi, agli aspetti demografici, alla sessualità, ecc.). In questa nuova dinamica il potere sovrano, che era stato tradizionalmente un potere di governo del territorio, è diventato un potere che mira al governo di tutto ciò che riguarda il bios. Per usare una terminologia schematica si può dire che il governo del territorio si è trasformato nel governo della popolazione.

Secondo questa ricostruzione, il paradigma classico del potere, rappresentato dalla sovranità, edificato intorno al diritto di vita e di morte di cui disponeva il pater familias nel

\footnotetext{
${ }^{36}$ M. Foucault, La volontà di sapere. Storia della sessualità, I [ed. or.: La volonté de savoir, Gallimard, Parigi, 1976], Feltrinelli, Roma, 2016, 122

${ }^{37}$ Duguit
} 
diritto romano, si è profondamente modificato proprio quando l'azione di governo ha iniziato a interessarsi alla vita. Il concetto di sovranità, spiega Foucault, è stato costruito sul paradigma del ius vitae ac necis di cui disponeva il pater familias nel diritto romano. Un potere sovrano che si fondava sulla morte, o meglio sul diritto di imporre la morte dei governati.

Come il pater familias esercitava il suo potere (assoluto) sovrano non solo sui beni ma anche su ciascun membro della propria famiglia, potendo legittimamente provocarne la morte, così il potere sovrano si è costruito proprio su quest'idea assoluta di potere che ci rimanda chiaramente l'immagine di un potere così intenso che può decidere della morte dei sudditi. Ma il potere restava estraneo al modo in cui i sudditi svolgevano la loro vita. Di qui un apparente paradosso perché il potere, mentre poteva determinare la morte dei sudditi, mandandoli a morire in guerra per la difesa della patria, per il resto si disinteressava del tutto delle loro vite, lasciandoli liberi di determinarsi liberamente in ogni altro ambito.

Scrive Foucault ${ }^{38}$ in un articolo dal significativo titolo «Diritto di morte e potere sulla vita» che l'esercizio della sovranità si caratterizzava «nel diritto di far morire o di lasciar vivere» ${ }^{39}$. Il potere, cioè, «era innanzitutto diritto di prendere: sulle cose, il tempo, corpi e infine la vita fino a culminare nel privilegio di impadronirsene per sopprimerla ${ }^{40}$. La massima manifestazione della autorità dello stato, in cui si evidenziava tutta la potenza dell'antico potere sovrano, era proprio nella possibilità del potere di disporre della vita dell'individuo, provocandone la morte.

Con la trasformazione del potere in senso biolitico si è assistito ad un vero e proprio capovolgimento del suo modo di manifestarsi, ciò che ha provocato l'irreversibile crisi della nozione di sovranità, categoria fondante il diritto pubblico dell'età moderna. Il nuovo potere biopolitico non si interessa più della difesa del territorio. Il suo oggetto è vita degli uomini in tutte le sue manifestazioni: salute, assetti demografici, bisogni alimentari, di sicurezza, di cura medica, ecc. «E ora sulla vita e lungo tutto il suo svolgimento - scrive Foucault - che il potere stabilisce la sua presa». Cambia pertanto il rapporto che il potere stesso ha con la morte, perché la morte diventa evidentemente il limite del potere, «il momento che gli sfugge». Così, in un senso solo apparentemente paradossale, il suicidio - che un tempo era considerato un crimine perché usurpare il diritto di mettere a morte in cui si aveva la massima manifestazione della sovranità statale, «sia diventato nel corso del diciannovesimo secolo uno dei primi comportamenti ad entrare nel campo dell'analisi sociologica; esso faceva apparire alle frontiere e negli interstizi del potere che si esercita sulla vita, il diritto individuale privato di morire» ${ }^{41}$.

Così è accaduto che il potere e la sua simbologia si sono allontanati dalla morte. In questo modo si spiega anche «la desuetudine» dei rituali che accompagnavano la morte. Ciò accadeva perché il momento della morte segnava simbolicamente il passaggio dalla sovranità terrestre a quella divina e «il fasto che la circondava partecipava della cerimonia politica» ${ }^{42}$, mentre ora il potere si allontana dalla morte perché essa ne segna il limite.

Il diritto di morire può quindi essere interpretato come una sorta di «replica politica»a tutte queste nuove e pervasive procedure di governo proprie del bio-potere ${ }^{43}$, per esercitare la fuga dalle procedure del potere biopolitico che controllano, dirigono, ordinano la vita, sino alle

\footnotetext{
${ }^{38}$ M. Foucault, La volontà di sapere. Storia della sessualità, 1, cit.

${ }^{39}$ Ibidem, 120

${ }^{40}$ Ibidem.

${ }^{41}$ Ibidem.

${ }^{42}$ Ibidem, 122.

${ }^{43}$ Ibidem, 123.
} 
sue fasi terminali. Insomma, per dirla con Foucault, «Quest'ostinazione a morire, così strana e tuttavia così regolare, così costante nelle sue manifestazioni, così poco spiegabile quindi con particolarità accidenti individuali, fu uno dei primi stupori di una società in cui il potere politico si era dato il compito di gestire la vita» ${ }^{44}$. Un diritto «incomprensibile per il sistema giuridico classico», che può essere interpretato come «la replica politica a tutte queste nuove procedure di potere che, a loro volta, non partecipano del diritto tradizionale della sovranità» ${ }^{45}$.

\section{LA MORTE NELLA FAMILIARITÀ DEI LUOGHI DOMESTICI}

Ma il diritto di morire assume un significato anche se letto in riferimento al modo con cui la società occidentale a noi contemporanea si relazione con la morte. Modo che non è stato sempre eguale. Non sempre, infatti, la morte ha destato le inquietudini che suscita attualmente nella società contemporanea. Per comprendere come un tempo l'atteggiamento di fronte alla morte fosse profondamente diverso da quello dei nostri giorni non occorre studiare l'argomento da una prospettiva antropologica. Vi sono, infatti, opere che della morte hanno tratteggiato la storia $^{46}$ e ve n'è traccia anche nella recente letteratura ${ }^{47}$ in cui ci sono testimonianze di come, persino in tempi a noi molto vicini, la morte ha continuato ad essere percepita in un modo profondamente diverso da quello attuale.

Per rendersi conto di questa diversità basta che si sposti il punto di vista da cui si osserva il fenomeno, abbandonando quello a noi oramai consueto delle grandi metropoli, per guardare quanto accadeva in un passato nemmeno lontanissimo, oppure anche quanto accade anche ai nostri giorni nei piccoli centri rurali, rimasti legati ad un diverso modo di accogliere la morte attraverso la conservazione, nella cultura popolare, di tutte quelle ritualità che per secoli hanno accompagnato il processo del morire, in cui l'intera collettività veniva coinvolta.

La diversità di atteggiamento nei confronti della morte esercitava la sua influenza anche sulle questioni di fine vita, rendendole meno drammatiche rispetto a quanto non lo siano attualmente. Uno sguardo su come era diverso l'atteggiamento nei confronti del morire può allora risultare utile a chi studia il fine vita dalla prospettiva dei principi costituzionali, perché ci svela un mondo in cui la partecipazione del malato, la sua volontà e il suo dominio consapevole sul processo del morire erano elementi centrali della regolazione che la società si era autonomamente data per la soluzione di queste vicende, non formalizzati in regole giuridiche, ma espressione del diffuso sentire e frutto, pertanto, del consenso sociale.

\footnotetext{
${ }^{44}$ Ibidem, 122.

${ }^{45}$ Ibidem, 128.

${ }^{46}$ ARIÈS, P., Storia della morte in Occidente: dal medioevo ai giorni nostri (ed or.: Essais sur l'histoire de la mort en occident: du Moyen Age à nos jours, 1975), Bur, Milano, 2013, I edizione digitale, passim; ELIAS, N., La solitudine del morente, Il Mulino, Bologna, 1985, passim. SPELLMANN, W.M., Breve storia della morte, BOLLATI BORINGHIERI, 2015, passim. "Le riflessioni che seguono riproducono, con opportune modifiche", argomentazioni svolte in un saggio pubblicato in DEL PIZZO, F. e GIUSTINIANI, P., Il biosistema tra tecnica ed etica. Nuove questioni di tecnica ambientale, Mimesis, Milano-Udine, 2018.

${ }^{47}$ Cfr. MURGIA. M., Accabadora, Einaudi, Torino, 2009, che offre molti spunti di riflessione sul tema della morte. Il quadro descritto in questo romanzo sorprende il cittadino di una metropoli contemporanea perché si vieni trascinati in un mondo che tratta le questioni legate alla morte in un modo completamente diverso rispetto a quello cui siamo abituati, in cui la morte è accolta con una sorprendente familiarità, cioè come qualcosa che rientra nella normalità della vita quotidiana. Ma vedi anche, sul tema specifico evocato dal libro, ossia sulla figura dell'accadadora, MURINEDDU, G., L'accabbadora. La morte invocata, Chronos 2007; TURCHI, D., Ho visto agire s'accabadora (la prima testimonianza oculare di una persona vivente sull'operato di s'accabadora), Edizioni Iris, 2008.
} 
Un tempo la morte era percepita in modo completamente diverso da quello cui siamo abituati. La morte era vissuta come un fenomeno sociale cui partecipava tutta la collettività, con manifestazioni di solidarietà che accompagnavano il morente e la sua famiglia a partire dal momento dell'insorgere della malattia sino al periodo del lutto.

Queste ritualità avevano la funzione di collettivizzare il dolore, circondando i cari dell'estinto con una rete di solidarietà collettiva che riusciva a rendere meno traumatica la inevitabile sofferenza per la scomparsa della persona amata e svolgeva anche una funzione pedagogica per le nuove generazioni, che vi prendevano parte.

Per avere un'idea di questo atteggiamento basta volgere lo sguardo lontano dalle grandi metropoli contemporanee della post modernità.

In un contesto che assomiglia molto di più alla società pre-industriale, anche se ambientato nella Sardegna del novecento, si svolge, ad esempio, il romanzo di Michela Murgia, L'accabadora ${ }^{48}$, che offre uno spaccato interessante e significativo di come una società rurale, che si caratterizza per un'economia agricola (in cui il lavoro manuale è il principale fattore produttivo e la difficoltà della vita si manifesta innanzitutto nella durezza del lavoro agricolo), si relaziona con le questioni legate all'inizio e al fine vita, senza mettere in scena tutta la drammaticità che turba profondamente il rapporto del cittadino contemporaneo con la morte.

Il quadro che ne emerge è che una società di questo tipo, vivendo in più diretto a contatto con la naturalità della vita, accoglie la malattia, il dolore, la sofferenza, il lutto, così come le emozioni e i sentimenti che ne derivano, riuscendo a stemperare la drammaticità che questi accadimenti portano con sé attraverso la loro collocazione in una dimensione che è allo stesso tempo pubblica ${ }^{49}$ e collettiva ${ }^{50}$. Proprio questa 'collettivizzazione' della sofferenza si concretizzava in un «sereno accompagnamento verso la fine da parte di un'intera comunità» ${ }^{51} \mathrm{e}$ svolgeva così la funzione di esorcizzare il dramma della morte e tutti i sentimenti forti ad essa legati. Il compimento di una ritualità ancestrale e collettiva aveva, insomma, la funzione di socializzare il dolore, riuscendo in questo modo a rendere più sopportabile tutto il carico di sofferenza che la morte, inevitabilmente, porta con sé.

Quanto descritto nel romanzo ricorda il modo di accogliere la morte descritto dagli studiosi che si sono dedicati alla storia della morte ${ }^{52}$ ed evoca anche le ritualità proprie delle culture tribali che accompagnano questo evento, come ad esempio, quelli delle tribù africane raccontati da Karen Blixen ${ }^{53}$, in cui la persona che sta per morire viene spostata appena fuori dalla sua capanna in modo da poter entrare in diretto contatto con tutta la collettività a lui più prossima. Scrive Karen Blixen: «al tempo in cui gli uomini degli altopiani erano liberi di morire come volevano, seguivano i costumi dei loro padri e delle loro madri. Quando un kikuyu si ammalava, la sua gente lo portavo fuori dalla sua capanna con il suo giaciglio di legni e pelli, poiché una capanna in cui era morto qualcuno non doveva più essere abitata ma doveva essere distrutta con il fuoco. Là fuori, sotto le alte fronde degli alberi, la sua famiglia sedeva intorno a

\footnotetext{
${ }^{48}$ Cfr. la nota che precede.

49 «La camera del moribondo si trasformava in un luogo pubblico», ARIÈS, P., Storia della morte in Occidente: dal medioevo ai giorni nostri, cit.

${ }^{50}$ «Era necessario che gli amici, i parenti, i vicini fossero presenti» ARIÈS, P., Storia della morte in Occidente: dal medioevo ai giorni nostri, cit.

${ }^{51}$ RODOTÀ, S., La vita e le regole, Feltrinelli, Milano, 2006, 261.

52 Cfr. ARIÈS, P., Storia della morte in Occidente: dal medioevo ai giorni nostri (ed or.: Essais sur l'histoire de la mort en occident: du Moyen Age à nos jours, 1975, cit., e gli altri autori citati alla nota 1.

${ }^{53}$ Pseudonimo della scrittrice danese Karen Christentze Dinesen,
} 
lui a tenergli compagnia, gli Squatter amici passavano a raccontargli le novità e i pettegolezzi della fattoria, la notte piccoli fuochi di carboni venivano accesi intorno al letto. Se il malato si ristabiliva veniva di nuovo portato nella capanna. Se moriva veniva trasportato al di là del fiume, nella pianura, e lasciato alla veloce e accurata opera di ripulitura degli avvoltoi, degli sciacalli, e dei leoni che scendevano dalle colline» ${ }^{54}$.

Nel passato, dunque, la morte, e tutto ciò che ad essa è associato, trovava una forma di espressione pubblica, diventando un fatto sociale cui partecipavano sia il morente, che era non solo cosciente della morte, ma ne era anche padrone ${ }^{55}$, e la comunità stessa.

Questo diverso modo di affrontare la morte è molto istruttivo per chi studia l'autodeterminazione del malato e i principi costituzionali inerenti al diritto alla salute e alle fasi terminali dell'esistenza.

In questi contesti, infatti, la partecipazione individuale del morente alle ritualità che riguardavano la malattia e la morte è un elemento avvertito come fondamentale. Innanzitutto, dunque, la morte non veniva nascosta al morente. Al contrario, il morente doveva essere consapevole del destino cui stava andando incontro. La consapevolezza della morte da parte del morente era ritenuta necessaria proprio a beneficio del morente stesso, perché egli potesse chiamare a sé i figli, fare tastamento, eventualmente chiedere scusa ai propri cari dei torti commessi, per congedarsi così nel modo più sereno possibile dal mondo ${ }^{56}$, in armonia con sé stesso e con i valori religiosi profondamente sentiti dalla società.

Paradossalmente, quello che gli uomini contemporanei generalmente si augurano pensando al momento della propria morte, cioè una morte improvvisa (come nel detto napoletano "a morte e' subbito"), quindi lontana dalla consapevolezza e dal proprio individuale dominio, era avvertita in tempi passati come la più grande sciagura che potesse capitare ad un uomo ${ }^{57}$.

Questa consapevolezza della propria morte assumeva un ruolo essenziale anche nell'ambito dei processi di fine vita i quali, sebbene non fossero oggetto di una disciplina formalizzata in regole giuridiche, erano interessati, comunque sia, da una forma di selfregulation socio-familiare ${ }^{58}$. Accogliere la morte come momento essenziale del processo naturale della vita non significava, dunque, che regnasse necessariamente l'assoluto dominio delle regole biologiche, ma significava consapevole accettazione del proprio destino e della malattia, della quale, tuttavia, in casi socialmente codificati, era - comunque sia - legittimo accelerare il processo ${ }^{59}$. La padronanza della morte da parte del morente e il ruolo attribuito alla

\footnotetext{
${ }^{54}$ BLIXEN, K., Il gran gesto, in Ombre sull'erba (1984), Adelphi, Milano, 1985, 73-74, riportato in RODOTÀ, S., La vita e le regole, cit., 261.

${ }^{55}$ Scrive ARIÈS, P., Storia della morte in Occidente: dal medioevo ai giorni nostri, cit, che la morte è una cerimonia pubblica «organizzata dal moribondo stesso, che la presiede e ne conosce il protocollo».

${ }^{56}$ ARIÈS, P., Storia della morte in Occidente: dal medioevo ai giorni nostri, cit.

${ }^{57}$ ARIÈS, P., Storia della morte in Occidente: dal medioevo ai giorni nostri, cit.

${ }^{58}$ La libera volontà del malato di porre fine alla sua agonia, come messo limpidamente in luce nel romanzo di Michela Murgia prima citato, rappresentava un elemento essenziale della regolazione sociale della morte. L'accabadora fugge sdegnata da una casa in cui l'avevano chiamata con la menzogna, cioè non per volontà di un malato ancora cosciente e quindi in grado di esprimere la sua volontà.

${ }^{59}$ La nascita e la morte, lungi dall'essere visti come processi naturali che si compivano da sé, erano percepiti come appartenenti allo stesso ordine di fenomeni, entrambi come processi in cui si doveva essere aiutati. Come il bambino viene aiutato a nascere, così il malato può essere aiutato a morire, finché è ancora cosciente, secondo la sua volontà individuale, che appare come la regola fondamentale della morte, cui la società armoniosamente si conforma, come se stesse agendo in base ad un diritto consuetudinario ancestrale «Quando la stessa donna aveva chiesto la grazia, le altre avevano agito per lei in un clima di condivisa naturalezza, dove atto illecito sarebbe parso piuttosto il non far nulla», scrive nel suo bel romanzo MURGIA, M., L'accabadora, cit.
} 
sua volontà (che domina questo processo) sorprende l'uomo contemporaneo, immerso in un dibattito sull'autodeterminazione che, per alcuni versi, pare arretrato rispetto a questa forma di regolazione sociale consuetudinaria, in cui l'esigenza che la volontà individuale sia la regola cardine di questo processo è in perfetta armonia con il sentire sociale.

Tutto questo produceva un atteggiamento di familiarità con la morte, molto lontano dal significato tragico e drammatico che oggi è ad essa associato, e in cui la morte è accolta «senza paura né disperazione, a metà strada fra la rassegnazione passiva e la fiducia mistica» ${ }^{60}$.

La consapevolezza del morente e la partecipazione collettiva rappresentavano un comportamento socialmente codificato di cui beneficiavano allo stesso tempo il morente e i suoi cari. «Attraverso la morte, ancor più che attraverso gli altri momenti critici dell'esistenza, il destino si rivela, e allora il morente lo accetta in una cerimonia pubblica il cui rito è fissato dalla consuetudine» ${ }^{61}$, scrive Philipe Ariès. La ritualità che si esprimeva nel lutto era una forma ancestrale di self-regulation che la società si era data per aiutare chi era colpito dalla malattia e $\mathrm{i}$ suoi cari ad affrontare il dolore provocato dalla morte e, così, ad elaborarlo e superarlo ${ }^{62}$.

Tutto ciò ci rappresenta un mondo in cui il fine vita è un processo naturale che resta confinato e regolato nell'ambiente domestico di cui sono protagonisti il morente stesso e la comunità a lui più vicina, sfuggendo al contempo al diritto positivo e al potere del medico.

\section{IL TABÙ DELLA MORTE E IL DOVERE DI ESSERE FELICI}

Nella dimensione moderna, e ancor più in quella post-moderna, la morte - invece - è vissuta come un vero e proprio tabù, cioè come un evento che deve essere rimosso $\mathrm{o}$, quantomeno, tenuto il più lontano possibile dalla quotidianità, il che significa non solo fuori dalle mura domestiche, ma anche dalla stessa possibilità di accoglierla nei discorsi e pensieri e di condividerla con la collettività. Come è stato opportunamente notato, nella società contemporanea si è assistito a una vera e propria «rimozione della morte a livello individuale e a livello sociale» ${ }^{63}$.

La fine dell'esistenza, pertanto, lungi dall'essere percepita come qualcosa appartenente all'ordine naturale delle cose è invece avvertita come qualcosa di inconcepibile, da esorcizzare, fino al punto di espellerla, allo stesso tempo, dalla vita sociale.

In questo modo, l'evento della morte, così come quello della malattia che normalmente

\footnotetext{
${ }^{60}$ Come osserva ARIÉS, P., Storia della morte in Occidente: dal medioevo ai giorni nostri, cit., quando descrive il rapporto che le società antiche avevano con il processo del morire.

${ }^{61}$ ARIÈS, P., «attraverso la morte, ancor più che attraverso gli altri momenti critici dell'esistenza, il destino si rivela, e allora il morente lo accetta in una cerimonia pubblica il cui rito è fissato dalla consuetudine»

${ }^{62}$ ARIÈS, P.

${ }^{63}$ ELIAS, N., La solitudine del morente, Il Mulino, Bologna, 1985, 27.
} 
Non è un caso, pertanto, che Geoffrey Gorer, nel 1955 abbia scritto un articolo dal significativo titolo The Pornography of death, osservando come nel XX secolo non solo la morte sia diventata un vero e proprio tabù, ma che essa abbia addirittura preso il posto di quello che è stato il più importante tabù della nostra civiltà, sin dai tempi antichissimi, ossia del tabù del sesso.

Goerer evidenzia molte similitudini tra il modo in cui la società si comportava di fronte alle questioni legate al sesso con quello in cui oggi si comporta di fronte al processo del morire $^{64}$. Così, ad esempio, mette in luce come un tempo ai bambini si raccontava che fossero nati sotto un cavolo, mentre era loro concesso di assistere a tutta la fase della morte di un proprio caro; adesso, al contrario, la menzogna è rivolta a celare la morte. Quando una famiglia è colpita da un lutto, ai bambini viene detto che il caro defunto riposa in un ameno luogo lontano, proprio in un periodo in cui, invece, sempre minori inibizioni si evidenziano nei confronti delle vicende legate alla sessualità.

Questa rimozione della morte e la conseguente solitudine che oramai caratterizza il processo del morire comportano anche un nuovo modo di vivere il dramma della malattia e il successivo dolore legato al lutto, diventati sentimenti che non si possono condividere con la comunità, che è meglio non siano manifestati pubblicamente, ma che restino all'interno della sfera più intima e individuale dell'esistenza, e confinati nel più ristretto circolo familiare possibile.

Per continuare con la metafora della pornografia della morte, si può rilevare che «Si piange da soli - scrive Gorer - in privato, così come ci si spoglia», cioè lontano dagli sguardi della gente, di nascosto, «come se il dolore fosse qualcosa di analogo alla masturbazione». La rimozione della morte e la sua espulsione dalla quotidianità è diventata, sempre secondo Gorer, «una patologia sociale».

Da esorcizzare è tutto ciò che è collegato al processo del morire, dalla malattia, alla morte, al dolore e alla sofferenza, tutte cose diventate impensabili, quasi fossero proibite, appunto, e pertanto bandite dalla stessa possibilità di espressione pubblica ${ }^{65}$.

La morte e il dolore sono così ricacciati nella sfera privatissima delle esistenze individuali, per essere celati agli occhi della società.

Da un punto di vista sociologico non è difficile comprendere quali siano le cause che sono alla base della trasformazione del modo in cui la società contemporanea si pone di fronte al dolore.

Una società che ha fatto della ricerca edonistica della felicità e del benessere, materialisticamente intesi, della cura del corpo e dei piaceri ad esso associati il proprio obiettivo, celebrando la giovinezza, la salute, il divertimento, come «idoli metafisici» ${ }^{66}$, e in cui tutto il sistema sociale è rivolto alla ricerca di quella che Pascal Bruckner ha definito «l'euphorie perpétuelle» ${ }^{67}$, finisce con il considerare la sofferenza come qualcosa di inconcepibile, ed è quindi espulsa dal discorso pubblico. Anzi, paradossalmente, può addirittura

\footnotetext{
${ }^{64}$ GORER, G., "The Pornography of death", in The Encounter, ott. 1955.

${ }^{65}$ BRUCKNER, P., L'euphorie perpetuelle. Essai sur le devoir de bonheur, Grasset, Parigi, 2000, 218

${ }^{66}$ BRUCKNER, P., op. cit., 219.

${ }^{67}$ La stessa felicità, come nota sempre BRUCKNER, P., sembra essere divenuta, paradossalmente, un «dovere», il sottotitolo del saggio di Brukner è appunto «le devoir de bonneur».
} 
essere giudicata alla stregua di un crimine, andando così oltre la metafora poco sopra evidenziata della pornografia ${ }^{68}$.

Se il dovere è quello di essere felici, se la vita sociale è tutta costruita sui cardini di salute, benessere e piaceri, allora non può stupire che la malattia possa essere considerata come un evento che può ricevere lo stesso trattamento sociale di un fatto antigiuridico, che deve svolgersi quindi in modo clandestino ${ }^{69}$. Lo stesso Brukner ricorda il romanzo ironico, ma incredibilmente previgente, di Samuel Butler, del 1872 che raccontava le vicende di un paese, Erhewon (anagramma della parola inglese nowhere), in cui la malattia era appunto punita come un crimine e il malato era considerato, per il fatto stesso di essere malato, un criminale. Scrive il giudice nella sentenza pronunciata nei confronti di un malato di tisi: «voi mi direte forse che non siete responsabile né della vostra nascita né della vostra educazione. Io risponderei che la vostra tisi, venga o meno da colpa vostra, è una colpa in voi e che il mio dovere è sorvegliare per proteggere la Repubblica da colpe di questo tipo. Voi potreste dirmi che per un infortunio siete un criminale ma io posso replicarvi che il vostro crimine è essere infortunato» ${ }^{70}$.

\section{LA SOLITUDINE DEL MORENTE TRA OSPEDALIZZAZIONE DELLA MORTE E POTERE MEDICO}

Il problema che si pone al giurista è quello di spiegare questo nuovo comportamento sociale di fronte alla morte e, soprattutto, di comprendere in che modo questa modifica dell'atteggiamento della società nei confronti della morte abbia avuto delle ripercussioni sul progressivo favore che si manifesta, tanto nella società, quanto negli ordinamenti giuridici, con riferimento al diritto di morire.

Un elemento centrale credo sia determinato dallo spostamento del luogo della morte dallo spazio familiare e quotidiano dell'appartamento privato al luogo pubblico degli ospedali.

È stato a tale proposito osservato che la prima negazione della morte nella nostra società è rappresentata dal suo allontanamento dalle mura domestiche. Paradossalmente, proprio nel momento in cui la morte esce dallo spazio 'privato' di queste mura per essere presa in carico dal servizio 'pubblico' sanitario, interrompe il suo legame con la collettività (in particolare, con la fratellanza e la solidarietà comunitarie). Al contrario, spostandosi negli ospedali, il processo del morire diventa pubblico in un senso molto diverso da quello, visto in precedenza, di collettivo: perché, uscendo dai luoghi della quotidianità, finisce per essere occultato allo sguardo della società, divenendo: da un lato, un fatto 'privato' del morente e dei suoi cari che lo gestiscono; dall'altro, un fatto sottoposto alla disciplina delle leggi dello stato e, in primo luogo, assoggettato alla regolazione del potere medico.

Scrive a questo proposito Stefano Rodotà che «la morte moderna viene identificata con il suo rifiuto sociale, che la espelle dai discorsi pronunciabili, dai contesti familiari, la medicalizza, la trasferisce negli ospedali, ne sottolinea gli aspetti di gestione economica» ${ }^{71}$.

Il paradosso è che questa medicalizzazione delle fasi terminali della vita finisce con il ripercuotersi sul morente, che del processo del morire perde consapevolezza e dominio,

\footnotetext{
${ }^{68}$ BRUCKNER, P., op. cit., 219.

${ }^{69}$ Di morte «clandestina» discorre SPELLMANN, W.M., Breve storia della morte, cit., 7.

${ }^{70}$ Citazione riportata da BRUCKNER, P., op. cit., 219. La responsabilità della traduzione è mia.

${ }^{71}$ RODOTÀ, S., La vita e le regole, cit., 260.
} 
trascinato in una condizione di emarginazione e di solitudine che viene vissuta come insopportabile. Osserva sempre Rodotà che «l'inclusione in un sistema che cresce le possibilità di cura, e quindi di sopravvivenza, si rovescia nell'esclusione del morente, sostanzialmente prigioniero dell'istituzione ospedaliera, alla quale viene affidata una delega sociale che si converte nell'allontanamento della morte dal discorso pubblico. Nel momento in cui il morire si trasferisce negli spazi pubblici si materializza quello che fa apparire un paradosso, l'occultamento della morte, che in realtà nasce dalla sua scomparsa dal quotidiano, dalla vita d'ogni giorno, da una sua gestione immersa nel flusso delle altre relazioni sociali, dal suo svolgersi negli stessi ambienti dove altrimenti si dorme, si fa l'amore. La morte viene allontanata dalla vita, se ne separa» ${ }^{72}$.

La morte medicalizzata perde così anche la sua dimensione naturale: diventa un processo artificiale in quanto completamente dominato dalla scienza medica. Da un lato, la potenza delle nuove tecnologie farmacologiche e rianimatorie riesce a protrarre per lungo tempo l'esistenza biologica della persona; dall'altro, all'opposto, il dato della naturalità abbandona la morte, in alcune circostanze ${ }^{73}$, con riferimento alla determinazione del suo momento, alla cui individuazione oramai concorre il sapere scientifico dei medici confortati dagli esami diagnostici. Sicché può accadere che, pur in presenza di attività cardiorespiratoria del morente, $\mathrm{i}$ medici dichiarino il soggetto clinicamente morto, ciò che pone non pochi problemi eticoreligiosi con riferimento, ad esempio, a chi crede che l'alito vitale sia la manifestazione con cui si esprime la divinità ${ }^{74}$, per cui è inconcepibile che la morte possa avvenire in un momento in cui la persona, comunque sia, respira ancora.

Scrive significativamente Ariè $^{75}$, a proposito di quanto appena osservato, che la morte è diventata «un fenomeno tecnico ottenuto con l'interruzione delle cure, cioè, in modo più o meno confessato, con una decisione del medico e dell'equipe ospedaliera. La morte è stata scomposta, frazionata in una serie di piccole tappe, di cui, in definitiva, non si sa quale sia la morte vera, quella in cui si è perduta la conoscenza, o quella in cui è venuto meno il respiro... tutte queste piccole morti silenziose hanno sostituito e cancellato la grande azione drammatica della morte, e nessuno ha più la forza o la pazienza di attendere per settimane un momento che ha perduto parte del suo significato».

La morte ospedalizzata, dunque, non solo esce dalla quotidianità della vita ma diventa anche un processo artificiale, a cui presiedere il potere medico. Il morente resta sempre più sullo sfondo, emarginato, solo. La sua consapevolezza e il suo dominio sul processo sono annichiliti, per cui ben si comprende come la sua reazione alla «prigione» dell'ospedale venga spesso affidata alla decisione, anch'essa solitaria, di porre fine ai trattamenti sanitari. Il diritto di morire, insomma, in questa prospettiva, finisce per essere interpretato piuttosto che come un'affermazione della propria autodeterminazione, come l'unica via di fuga possibile da questa nuova dimensione solitaria della morte.

Il luogo in cui si muore, casa od ospedale, assume un ruolo importante. Questo aspetto del problema, che pare oggi sottovalutato, dovrebbe invece spiegare un'importante influenza nell'attuale dibattito etico e giuridico intorno al fine vita e all'autodeterminazione del malato.

\footnotetext{
${ }^{72}$ RODOTÀ, S., La vita e le regole, cit., 261

${ }^{73}$ C'è infatti la morte artificiale, imposta dalla necessità dei trapianti d'organo, che può entrare in contrasto con la libertà di coscienza individuale.

${ }^{74}$ CASONATO, C., Introduzione al bio-diritto. La bioetica nel diritto costituzionale e comparato, Università degli Studi di Trento, 2006, 19 e ss.

${ }^{75}$ ARIÈS, P., Storia della morte, cit.
} 
Lo sforzo del legislatore dovrebbe pertanto tendere a riumanizzare la morte, riconducendola nei luoghi in cui si essa si è svolta per secoli, cioè nei luoghi degli affetti privati, aiutando così la società a ridarle quel significato collettivo che ha avuto nel passato, che contribuiva ad esorcizzarla e a renderla meno drammatica. Un passo in questa direzione è stato certamente compiuto con la legge n. 38 del 2010, recante «Disposizioni per garantire l'accesso alle cure palliative e alla terapia del dolore», nella parte in cui organizza il sistema sanitario in modo che possa intervenire per offrire le terapie ivi previste con assistenza domiciliare. Ovviamente molti passi ancor dovranno essere fatti in sede di attuazione di questa legge per fare uscire il morente dalla sua solitudine, che significa non solo ricondurre la morte nei luoghi degli affetti familiari, ma anche riattribuire al morente il pieno dominio della fine della sua vita ${ }^{76}$.

A conclusione di quanto esposto, si possono formulare alcuni spunti di riflessione.

Innanzitutto, il diritto di morire, per il quale si individua, correttamene a mio avviso, un chiaro riconoscimento costituzionale, assume una connotazione molto particolare. Piuttosto che leggerlo come un diritto paradossale perché annulla ogni altro diritto (in quanto può essere esercitato una sola volta), in realtà si configura come il diritto che consente di sottrarsi al controllo individuale, e sempre più pervasivo, esercitato dai nuovi dispositivi di potere (nel nostro caso del potere medico) cui è affidata la gestione delle fasi terminali della vita, che tiene in ostaggio un corpo esanime, imprigionandolo nell'ospedale. Piuttosto che un diritto per affermare la libertà individuale, si tratta di un diritto di fuga dal sistema di controllo pervasivo della vita e al dominio del potere medico, che si esercita anche sulle fasi terminali dell'esistenza.

In secondo luogo può osservarsi che l'affermazione del diritto di rifiutare le cure fino a lasciarsi morire, così come del diritto di morire nel senso di chiedere di essere aiutati a morire (anche se interpretati come manifestazione della preminenza dell'autodeterminazione del malato sul proprio corpo, sulla propria salute e quindi anche sulla propria vita), sono tutte 'conquiste' che non riescono ad eliminare l'aspetto più crudele del fine vita nella società moderna, che è quello della solitudine del morente, che è conseguenza, come osservato, dell'espulsione della morte dallo spazio collettivo e sociale.

Anzi, all'opposto, l'affermazione di questo diritto non incide sulla dimensione solitaria del morente, perché il morente, consapevole del rifiuto sociale della morte, marginalizzato in un luogo di cura, fuori dal contesto familiare in cui ha vissuto tutta la sua esistenza, sente ancora più forte la propria condizione di emarginazione e di solitudine. Altro discorso sarebbe attribuire nuovamente al morente il pieno controllo delle fasi terminali della sua vita nell'ambito del suo contesto familiare e comunitario.

Proprio la comunità degli affetti, dunque, mentre un tempo interpretava un ruolo di coprotagonista nel processo che accompagnava il malato alla morte, adesso, al contrario, si eclissa lasciando il morente solo con se stesso: relegandolo nelle istituzioni di cura fa aumentare la sua solitudine, contribuendo a costruire il contesto su cui si fonda la richiesta di terminare la vita, facendola apparire, appunto, una strada di fuga.

È innegabile, infatti, che in una società in cui il valore della solidarietà è diventato recessivo, mentre sono in esponenziale espansione i principi della concorrenza e della competitività assieme alla ricerca edonistica del piacere, influenza oggettivamente il malato, portato a sentirsi sempre più solo ed espulso dalla vita collettiva, anche da quella familiare, incentivando così, indirettamente, la sua scelta, sempre più solitaria, verso la fine della vita.

\footnotetext{
${ }^{76}$ RODOTÀ, S., La vita e le regole, cit., 262.
} 